\title{
ERRATA
}

\section{ERRATUM TO: SYNTHESIS, CHARACTERIZATION, COMPUTATIONAL STUDIES AND SINGLE CRYSTAL STRUCTURES OF $\left[\mathrm{Ru}(\mathrm{N}-\mathrm{P})_{2}(\mathrm{O}-\mathrm{O})\right]$ COMPLEXES}

\author{
M. A. Al-Wahish ${ }^{1}$, F. F. Awwadi ${ }^{1}$, and \\ H. A. Hodali ${ }^{1 *}$
}

DOI: $10.1134 / \mathrm{S} 0022476621090183$

In the original article there was a mistake in the Table 1 . The correct Table 1 is:

TABLE 1. Crystal Data and Structure Refinement for the Ruthenium Complexes 1, 2 and 3

\begin{tabular}{|c|c|c|c|}
\hline Parameter & Complex 1 & Complex 2 & Complex 3 \\
\hline Empirical formula & $\mathrm{C}_{44} \mathrm{H}_{32} \mathrm{~N}_{2} \mathrm{O}_{4} \mathrm{P}_{2} \mathrm{Ru}$ & $\mathrm{C}_{45} \mathrm{H}_{34} \mathrm{~N}_{2} \mathrm{O}_{4} \mathrm{P}_{2} \mathrm{Ru} \cdot 2 \mathrm{H}_{2} \mathrm{O}$ & $\mathrm{C}_{48} \mathrm{H}_{38} \mathrm{~N}_{2} \mathrm{O}_{4} \mathrm{P}_{2} \mathrm{Ru}$ \\
\hline Formula weight & 815.72 & 865.78 & 869.81 \\
\hline Temperature, $\mathrm{K}$ & $293(2)$ & 293(2) & 293(2) \\
\hline Crystal system & Triclinic & Triclinic & Monoclinic \\
\hline Space group & $P \overline{1}$ & $P \overline{1}$ & $P 2_{1} / c$ \\
\hline$a, b, c, \AA$ & $\begin{array}{c}10.7215(11), 11.0719(12) \\
16.6297(17)\end{array}$ & $\begin{array}{c}12.5358(5), 12.6559(5), \\
13.7056(6)\end{array}$ & $\begin{array}{c}14.4833(4), 11.9329(3), \\
24.7857(6)\end{array}$ \\
\hline$\alpha, \beta, \gamma, \operatorname{deg}$ & $\begin{array}{c}\text { 71.701(9), 83.791(9), } \\
74.889(9)\end{array}$ & $\begin{array}{c}90.514(3), 104.870(4) \\
90.540(3)\end{array}$ & 90, 97.438(2), 90 \\
\hline$V, \AA^{3}$ & $1808.7(3)$ & $2101.36(15)$ & $4247.60(19)$ \\
\hline Z & 2 & 2 & 4 \\
\hline$\mu, \mathrm{mm}^{-1}$ & 0.570 & 0.498 & 0.490 \\
\hline $2 \theta$ range for data collection, deg & 6.106 to 52.598 & 6.44 to 52.596 & 5.756 to 52.598 \\
\hline Index ranges & $\begin{array}{l}-13 \leq h \leq 13 \\
-13 \leq k \leq 18 \\
-20 \leq l \leq 20\end{array}$ & $\begin{array}{l}-17 \leq h \leq 17 \\
-17 \leq k \leq 18 \\
-17 \leq l \leq 18\end{array}$ & $\begin{array}{l}-15 \leq h \leq 18 \\
-12 \leq k \leq 14 \\
-30 \leq l \leq 30\end{array}$ \\
\hline Reflections collected / independent & 11452 / 6770 & $17812 / 8510$ & 21333 / 8609 \\
\hline$R_{\text {int }}, R_{\sigma}$ & $0.0333,0.0776$ & $0.0293,0.0562$ & $0.0317,0.0513$ \\
\hline Data / restraints / parameters & $6770 / 0 / 478$ & $8510 / 1 / 511$ & 8609 / 0 / 524 \\
\hline$G O O F$ on $F^{2}$ & 1.025 & 1.049 & 1.024 \\
\hline Final $R$ indexes $(I \geq 2 \sigma(I))$ & $\begin{array}{c}R_{1}=0.0456 \\
w R_{2}=0.0939\end{array}$ & $\begin{array}{c}R_{1}=0.0349 \\
w R_{2}=0.0780\end{array}$ & $\begin{array}{c}R_{1}=0.0382 \\
w R_{2}=0.0761\end{array}$ \\
\hline Final $R$ indexes (all data) & $\begin{array}{l}R_{1}=0.0680 \\
w R_{2}=0.1033\end{array}$ & $\begin{array}{l}R_{1}=0.0468 \\
w R_{2}=0.0825\end{array}$ & $\begin{array}{l}R_{1}=0.0567 \\
w R_{2}=0.0864\end{array}$ \\
\hline Largest diff. peak / hole, e $/ \AA^{3}$ & $0.70 /-0.87$ & $0.54 /-0.40$ & $0.44 /-0.37$ \\
\hline CCDC number & 2033145 & 2033176 & 2033175 \\
\hline
\end{tabular}

${ }^{1}$ Department of Chemistry, Faculty of Science, The University of Jordan, Amman, Hashemite Kingdom of Jordan; *h-hodali@ju.edu.jo. Original article submitted November 15, 2020; revised December 23, 2020; accepted December 28, 2020 . 
In the text of the article on the page 1057, instead of "di-(phenylphosphino)methane" should read as "bis(diphenylphosphino)methane"; on the page 1060 instead "were calculated using time" should read as "were calculated using TD-DFT"; on the page 1061 instead "8-(diphenylphosphino)quinolone" should read as "8-(diphenylphosphino)quinoline".

The original article can be found online at https://doi.org/10.1134/S002247662107009X. 